This item was submitted to Loughborough's Research Repository by the author.

Items in Figshare are protected by copyright, with all rights reserved, unless otherwise indicated.

\title{
Trapping of waves by a submerged elliptical torus
}

PLEASE CITE THE PUBLISHED VERSION

http://jfm-www.damtp.cam.ac.uk/

LICENCE

CC BY-NC-ND 4.0

REPOSITORY RECORD

Mclver, M., and R. Porter. 2019. "Trapping of Waves by a Submerged Elliptical Torus". figshare. https://hdl.handle.net/2134/694. 


\title{
Trapping of waves by a submerged elliptical torus
}

\author{
by M. McIver* \& R. Porter ${ }^{\dagger}$ \\ * Department of Mathematical Sciences, Loughborough University, Loughborough, Leicestershire, LE11 \\ $3 \mathrm{TU}, \mathrm{UK}$ \\ † School of Mathematics, University of Bristol, University Walk, Bristol, BS8 1TW, UK
}

\begin{abstract}
An investigation is made into the trapping of surface gravity waves by totally submerged three-dimensional obstacles and strong numerical evidence of the existence of trapped modes is presented. The specific geometry considered is a submerged elliptical torus. The depth of submergence of the torus and the aspect ratio of its cross-section are held fixed and a search for a trapped mode is made in the parameter space formed by varying the radius of the torus and the frequency. A plane wave approximation to the location of the mode in this plane is derived and an integral equation and a side condition for the exact trapped mode are obtained. Each of these conditions is satisfied on a different line in the plane and the point at which the lines cross corresponds to a trapped mode. Although it is not possible to locate this point exactly, because of numerical error, existence of the mode may be inferred with confidence as small changes in the numerical results do not alter the fact that the lines cross.

If the torus makes small vertical oscillations, it is customary to try and express the fluid velocity as the gradient of the so-called heave potential, which is assumed to have the same time dependence as the body oscillations. A necessary condition for the existence of this potential at the trapped mode frequency is derived and numerical evidence is cited which shows that this condition is not satisfied for an elliptical torus. Calculations of the heave potential for such a torus are made over a range of frequencies, and it is shown that the force coefficients behave in a singular fashion in the vicinity of the trapped mode frequency. An analysis of the time domain problem for a torus which is forced to make small vertical oscillations at the trapped mode frequency shows that the potential contains a term which represents a growing oscillation.
\end{abstract}

\section{Introduction}

In linear water-wave theory a trapped mode is defined to be a non-zero solution of the homogeneous equations and boundary conditions which doesn't radiate waves and has finite energy. If waves 
may be trapped by a particular body or system of bodies at a certain frequency then this means that the solution to any forced problem of this system is not unique at that frequency. Several bodies do not trap modes at any frequencies and a review of the classes of obstacles for which the forced boundary value problem has a unique solution is given in McIver (1996). However in that work a pair of two-dimensional surface-piercing obstacles was constructed which trap a wave at a certain frequency. The mode was constructed from a combination of point sources, placed so that there is no radiation of waves to infinity. The bodies were then defined to be portions of a pair of the streamlines of the flow which removed the singularities from the fluid. A similar construction of a floating toroid which supports a trapped mode was made by McIver \& McIver (1997) with the use of an axisymmetric potential and its Stokes' stream function. Calculations were made of the heave added mass and damping of the toroid by Newman (1999), and these support the existence of a trapped mode as the force coefficients behave in a singular manner around the trapped mode frequency.

Numerical evidence of further trapped modes was supplied by Evans \& Porter (1998) who showed that the potential due to two sources suitably placed in the free surface in the neighbourhood of a submerged circular cylinder could also be interpreted as a mode which is trapped by two surface-piercing cylinders next to a submerged circular cylinder. Kuznetsov \& McIver (1997) also extended the work of McIver \& McIver (1997) to generate numerically non-axisymmetric trapped modes which are supported by axisymmetric structures. More recently McIver \& Newman (2001) have shown that a non-axisymmetric structure may trap a wave whose flow field is independent of the azimuthal angle. The first numerical example of waves trapped by totally submerged bodies was given by McIver (2000) who used a similar procedure to that in McIver (1996) to construct a pair of bodies from the streamlines associated with submerged dipoles. It is much more difficult to construct submerged bodies in this way because it is necessary to show that two of the streamlines of the flow are closed. It follows that the trapped modes constructed are in some sense more unstable than those associated with surface-piercing bodies as there is only one pair of streamlines that may be chosen to represent the bodies. Simon \& Ursell (1984) show that trapped modes can not exist for any system of bodies which are submerged in deep water and which are contained between lines which intersect the free surface at the same point and at angle of $45^{\circ}$ to the horizontal. They speculated that it might be possible to decrease this angle, but the fact that trapped modes exist for some submerged bodies means that the angle cannot be reduced to zero.

Evans \& Porter (1998) gave an argument that suggested that trapped modes may be constructed for pairs of identical two-dimensional bodies which have the property that either of the 
bodies separately has a zero of transmission at a certain frequency. If the bodies are placed a suitable distance apart, under a wide spacing approximation waves may be reflected backwards and forwards between the obstacles and the motion is effectively trapped between the pair. Linton \& Kuznetsov (1997) applied this idea to give numerical evidence of trapped modes between pairs of surface-piercing angled barriers and more recently Kuznetsov, McIver \& Linton (2001) have provided similar evidence of wave trapping by four vertical surface-piercing barriers. Porter (2001a) has also shown that zeros of transmission are possible for certain families of submerged bodies, which include ellipses. In further work (Porter 2001b) he went on to give strong numerical evidence for the existence of trapped modes for pairs of such ellipses, separated by an amount which is close to that predicted by the wide spacing approximation.

To date there have been no examples of three-dimensional submerged bodies which trap waves. However, the argument that pairs of two-dimensional obstacles which separately possess zeros of transmission may support trapped modes may be extended to a three-dimensional torus with the use of the plane wave approximation of Simon (1982). The details of this argument are presented in the next section. However this argument only leads to an approximation to a trapped mode and it cannot be used to conclude that genuine trapped modes exist. In section 3 the method of Porter (2001b) is followed and an integral equation is derived which an exact trapped mode potential must satisfy. After the solution of the integral equation is approximated by a Fourier series, a trapped mode frequency is shown to correspond to a zero eigenvalue of a real matrix, provided that the corresponding eigenfunction satisfies a side condition. Numerical results are presented in section 4 which suggest that trapped modes do exist for certain submerged toruses and it is shown that the shape of the torus and the value of the trapped mode frequency are close to the values predicted by the plane wave approximation. Calculations are made of the solution to the forced heave problem and it is shown that the force coefficients behave in a singular fashion in the vicinity of the trapped mode frequency. An analysis of the time domain problem for a torus which is forced to make small vertical oscillations at the trapped mode frequency shows that the potential contains a term which represents a growing oscillation.

\section{The plane wave approximation}

A two-dimensional body, which is totally submerged in deep water, is rotated through $360^{\circ}$ about a vertical axis outside the body, to form a submerged torus. (A vertical cross-section through an elliptical torus is given in figure 1.) The axis of rotation is at a distance $c$ from a polar reference 


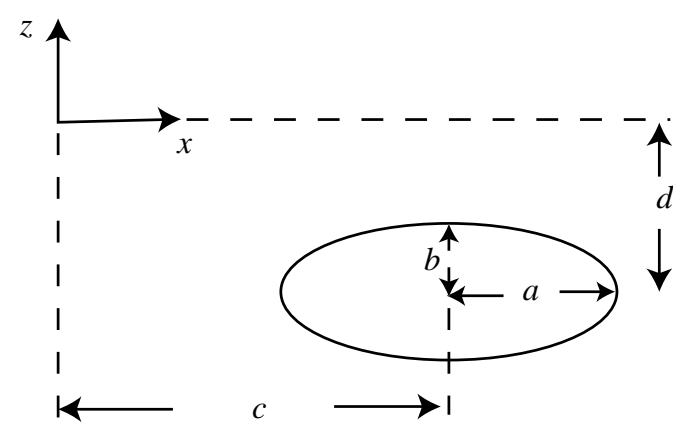

Figure 1: A vertical cross-section through a submerged elliptical torus

axis through the body where $c / a \gg 1$ and $a$ is a typical dimension of the original two-dimensional body. Rectangular Cartesian coordinates are chosen so that the origin is in the mean free surface above the centre of the torus and the $z$-axis points vertically upwards. The fluid is assumed to be inviscid and incompressible and the motion is irrotational. Thus the velocity is given by the gradient of a potential which satisfies Laplace's equation. Trapped modes are defined to be oscillations of the fluid which satisfy the linearised free surface boundary conditions, have zero normal velocity on the surface of the body, decay to zero at large depths and which have finite energy. Thus, an axisymmetric trapped mode potential is given by $\operatorname{Re}\left[\phi(r, z) \mathrm{e}^{-\mathrm{i} \omega t}\right]$, where $\omega$ is the angular frequency of oscillation of the mode and $r^{2}=x^{2}+y^{2}$. The potential $\phi$ satisfies

$$
\nabla^{2} \phi=0
$$

in the fluid domain, $\mathcal{D}$,

$$
\begin{gathered}
K \phi-\phi_{z}=0, \quad \text { on } z=0, \\
\phi \rightarrow 0, \quad \text { as } \quad z \rightarrow-\infty
\end{gathered}
$$

and

$$
\frac{\partial \phi}{\partial n}=0, \quad \text { on } S,
$$

where $S$ is the surface of the torus, $K=\omega^{2} / g, g$ is the acceleration due to gravity and $K c \gg 1$. The derivative $\partial / \partial n$ is in the inward normal direction to $S$. Finally, in order for the mode to have finite energy it is necessary that

$$
\iiint_{\mathcal{D}}|\nabla \phi|^{2} \mathrm{~d} x \mathrm{~d} y \mathrm{~d} z+K \iint_{z=0}|\phi|^{2} \mathrm{~d} x \mathrm{~d} y<\infty .
$$

A consequence of this last condition is that there are no waves at infinity, that is

$$
\phi=o\left(r^{-\frac{1}{2}}\right), \quad \text { as } r \rightarrow \infty .
$$


The method of matched asymptotic expansions is used to obtain an approximation to $\phi$. A potential is sought which represents a standing wave in the region $r \ll c$ and is wavefree for $r \gg c$. In the vicinity of the torus, the governing equation for $\phi$ is shown to reduce to the twodimensional Laplace equation in the presence of a submerged obstacle $B$, which is formed from the cross-section of the torus. In order to be able to match the potentials, the outer limit of the solution for $\phi$ in the interior of the torus must represent a plane wave which is totally reflected by the body B. (A similar procedure was used by Newman (1977) to determine the radiation and scattering of waves by a floating slender torus.)

In the interior of the torus, the axisymmetric standing wave is given by

$$
\phi=J_{0}(K r) \mathrm{e}^{K z}
$$

where $J_{0}$ is the zero-order Bessel function of the first kind. The plane wave approximation developed by Simon (1982) is used to approximate this potential for large $K c$ in the vicinity of the torus, where $K(r-c)=O(1)$. Thus $K r$ is written as $K r=K(r-c)+K c$ and the addition theorem and large argument expansion of Bessel functions (9.1.75 and 9.2.1 respectively in Abramowitz \& Stegun (1965) ) are used to write

$$
\begin{aligned}
J_{0}(K r) & =\sum_{n=-\infty}^{\infty} J_{-n}(K(r-c)) J_{n}(K c) \\
& \sim \sqrt{\frac{2}{\pi K c}} \sum_{n=-\infty}^{\infty} J_{-n}(K(r-c)) \cos \left(K c-\frac{n \pi}{2}-\frac{\pi}{4}\right), \quad \text { as } \quad K c \rightarrow \infty
\end{aligned}
$$

where $J_{n}$ is the $n$ th-order Bessel function of the first kind. The results

$$
\sum_{n=-\infty}^{\infty} \mathrm{i}^{n} J_{n}(K(r-c))=\mathrm{e}^{\mathrm{i} K(r-c)} \quad \text { and } \quad \sum_{n=-\infty}^{\infty}(-\mathrm{i})^{n} J_{n}(K(r-c))=\mathrm{e}^{-\mathrm{i} K(r-c)}
$$

may be obtained in a straightforward fashion from (9.1.42) and (9.1.43) in Abramowitz \& Stegun (1965), and after some manipulation they may be used to show that

$$
\phi \sim \sqrt{\frac{1}{2 \pi K c}} \mathrm{e}^{\mathrm{i}(K c-\pi / 4)+K z}\left[\mathrm{e}^{\mathrm{i} K(r-c)}+\mathrm{ie}^{-2 \mathrm{i} K c} \mathrm{e}^{-\mathrm{i} K(r-c)}\right], \quad K c \gg 1, \quad K(r-c)=O(1) .
$$

In the vicinity of the body of the torus, $\phi$ and its derivatives vary on the length scale $a$ but $r=O(c)$ and so, to a first approximation, the term $\phi_{r} / r$ may be neglected in Laplace's equation. Thus

$$
\nabla^{2} \phi \approx \phi_{r r}+\phi_{z z}
$$

which means that $\phi$ satisfies the two-dimensional Laplace's equation in a plane of constant azimuthal angle. As the boundary conditions are unchanged in this region, the potential represents the scattering of two-dimensional plane waves by the cross-section of the torus $B$. 
There are no waves exterior to the torus and so the outer limits of the interior potential near the torus in (8) show that $\phi$ represents a plane wave which propagates outwards along a radial line and which is totally reflected by the body $B$. Clearly this can only occur if the wave number corresponds to a value at which there is zero transmission of waves across $B$ and if the reflection coefficient is given by

$$
R=\mathrm{ie}^{-2 \mathrm{i} K c} .
$$

This last equation gives possible values of $K c$ in terms of the phase of the reflection coefficient. So, if $R=\mathrm{e}^{\mathrm{i} \delta}$ is known from the solution of the two-dimensional scattering problem for $B$, then

$$
K c=\frac{\pi}{4}-\frac{\delta}{2}+n \pi
$$

where $n$ is an arbitrary integer. It is interesting to observe that the corresponding result for a wide-spacing approximation to two-dimensional modes trapped between two bodies, which are

separated by a distance $c$, is $K c=-\delta / 2+n \pi$, and so there is a difference of $\pi / 4$ between the two and three dimensional approximate values of $K c$.

\section{Integral equation formulation}

The analysis of the previous section is only approximate and so it is not obvious whether a torus can support trapped modes or whether it just has highly tuned resonances. In this section the method of Porter (2001b) is followed and a system of integral equations is constructed, whose solution corresponds to an axisymmetric trapped mode for a submerged torus of arbitrary crosssection. An investigation of the existence of such solutions will be made in a later section and it will be shown that there is a perturbation of a torus which is predicted to support trapped modes by the plane wave approximation which supports actual trapped modes.

The boundary of the cross-section of the torus $B$ is denoted by $C$ and is parametrised by

$$
(r, z)=(R(t), Z(t))=\left(c+C_{r}(t),-d+C_{z}(t)\right), \quad 0 \leq t<2 \pi
$$

where $t$ is measured anticlockwise from the radial line outwards from the point $(r, z)=(c,-d)$. Thus $d$ is a measure of the depth of submergence of the torus and, to ensure that the torus is completely submerged, $C_{z}(t)<d, 0 \leq t<2 \pi$.

Since the equations and boundary conditions which define $\phi$ are homogeneous, $\phi$ may be taken to be real without any loss of generality. The basic ingredient in the formulation of the integral 
equations is the Green's function for a uniform ring source submerged a distance $\left|z_{0}\right|$ below the free surface with a radius $r_{0}$. The Green's function, $\operatorname{Re}\left[G\left(r, z ; r_{0}, z_{0}\right) \mathrm{e}^{-\mathrm{i} \omega t}\right]$, which has no azimuthal variation is the solution of the equation

$$
\nabla^{2} G\left(r, z ; r_{0}, z_{0}\right)=-2 \pi r_{0} \delta\left(z-z_{0}\right) \delta\left(r-r_{0}\right), \quad \text { for } z, z_{0}<0
$$

and, for $\rho=\left\{\left(r-r_{0}\right)^{2}+\left(z-z_{0}\right)^{2}\right\}^{\frac{1}{2}} \neq 0$, satisfies the boundary conditions (2) and (3). It may be shown that

$$
G\left(r, z ; r_{0}, z_{0}\right) \sim-\frac{1}{2 \pi} \ln K \rho, \quad \text { as } K \rho \rightarrow 0 .
$$

and so $G$ exhibits a source-like behaviour which is similar to a purely two-dimensional line source.

Hulme (1983) showed that the Green's function which satisfies the above equations is given by

$$
\begin{aligned}
& G\left(r, z ; r_{0}, z_{0}\right)=\mathrm{i} \pi K r_{0} \mathrm{e}^{K\left(z+z_{0}\right)} J_{0}\left(K r_{<}\right) H_{0}\left(K r_{>}\right) \\
& +\frac{2 K r_{0}}{\pi} \int_{0}^{\infty}(\nu \cos \nu K z+\sin \nu K z)\left(\nu \cos \nu K z_{0}+\sin \nu K z_{0}\right) I_{0}\left(\nu K r_{<}\right) K_{0}\left(\nu K r_{>}\right) \frac{\mathrm{d} \nu}{\nu^{2}+1}(15
\end{aligned}
$$

where

$$
r_{<}=\min \left\{r, r_{0}\right\}, \quad r_{>}=\max \left\{r, r_{0}\right\}
$$

and $J_{0}, I_{0}, K_{0}$ and $H_{0} \equiv J_{0}+\mathrm{i} Y_{0}$ are Bessel functions of zeroth order. Far away from the ring source, the Green's function generates outgoing circular waves, which are characterised by the behaviour

$$
G\left(r, z ; r_{0}, z_{0}\right) \sim \mathrm{i} K r_{0} \mathrm{e}^{K\left(z+z_{0}\right)} J_{0}\left(K r_{0}\right) \sqrt{\frac{2 \pi}{K r}} \mathrm{e}^{\mathrm{i}(K r-\pi / 4)}, \quad \text { as } K r \rightarrow \infty .
$$

An application of Green's identity to $\phi$ and $G$ in the domain $\mathcal{D}$ gives

$$
-r_{0} \phi\left(r_{0}, z_{0}\right)=\int_{C} r \phi(r, z) \frac{\partial}{\partial n} G\left(r, z ; r_{0}, z_{0}\right) \mathrm{d} s, \quad\left(r_{0}, z_{0}\right) \notin C,
$$

where the governing equations for $\phi$ and $G$ and their boundary conditions (1) - (4) and (13) have been used, and the azimuthal integration has been performed explicitly ( $s$ measures the arc length along the curve $C$ ).

An integral equation of the second kind defining $\phi$ on the curve $C$ can be found by moving the point $\left(r_{0}, z_{0}\right)$ onto $C$, when a factor of $\frac{1}{2}$ multiplies the left hand side of (18). Trapped modes then correspond to non-trivial functions $\phi$ which satisfy this complex-valued equation. However computational difficulties can arise when using (18) for slender bodies, due to the singular nature of the kernel. Instead, the parametrisation introduced at the beginning of this section is used to write

$$
\frac{\partial}{\partial n}=\frac{1}{\sigma(t)}\left(-Z^{\prime}(t) \frac{\partial}{\partial r}+R^{\prime}(t) \frac{\partial}{\partial z}\right)
$$


on the curve $C$, where the primes in this context denote derivatives with respect to the argument, whilst the tangential derivative on the curve $C$ in the direction of $t$ increasing is given by

$$
\frac{\partial}{\partial s}=\frac{1}{\sigma(t)}\left(Z^{\prime}(t) \frac{\partial}{\partial z}+R^{\prime}(t) \frac{\partial}{\partial r}\right)
$$

Here,

$$
\sigma(t)=\sqrt{\left(R^{\prime}(t)\right)^{2}+\left(Z^{\prime}(t)\right)^{2}}
$$

is a normalising factor. Not only are we going to use (19) and (20) as representations for the normal and tangential derivatives for points on $C$, but also for points away from $C$. The derivative $\partial / \partial n_{0}$ of (18) is taken, according to the definition in (19), to give

$$
\frac{\partial}{\partial n_{0}} \phi\left(r_{0}, z_{0}\right)=-\frac{\partial}{\partial n_{0}} \int_{C} r \phi(r, z) \frac{1}{r_{0}} \frac{\partial}{\partial n} G\left(r, z ; r_{0}, z_{0}\right) \mathrm{d} s, \quad\left(r_{0}, z_{0}\right) \notin C .
$$

Since the fluid motion is axisymmetric, there is a Stokes stream function, $\psi$, for the flow, which is related to the potential by

$$
\frac{\partial \phi}{\partial r}=\frac{1}{r} \frac{\partial \psi}{\partial z}, \quad \frac{\partial \phi}{\partial z}=-\frac{1}{r} \frac{\partial \psi}{\partial r}
$$

These relations may be combined with (19) and (20) to show that

$$
\frac{\partial \phi}{\partial n}=-\frac{1}{r} \frac{\partial \psi}{\partial s}
$$

The Stokes stream function is constant on the surface of a solid boundary. The stream function may be defined to within an arbitrary additive constant and so we adopt convention and define

$$
\psi(r, z) \rightarrow 0, \quad \text { as }\left(z^{2}+r^{2}\right) \rightarrow \infty
$$

so that

$$
\psi(r, z)=\psi_{C}, \quad \text { for }(r, z) \in C
$$

for some unknown value $\psi_{C}$. Also, it is not difficult to confirm, using standard results for Bessel functions, that

$$
\frac{\partial}{\partial n_{0}}\left(\frac{1}{r_{0}} \frac{\partial G}{\partial n}\right)=-\frac{1}{r_{0}} \frac{\partial}{\partial s_{0}}\left(\frac{1}{r} \frac{\partial H}{\partial s}\right)
$$

where

$$
\begin{aligned}
& H\left(r, z ; r_{0}, z_{0}\right)=-\mathrm{i} \pi K r K r_{0} \mathrm{e}^{K\left(z+z_{0}\right)} J_{1}\left(K r_{<}\right) H_{1}\left(K r_{>}\right) \\
& +\frac{2 K r K r_{0}}{\pi} \int_{0}^{\infty}(\nu \sin \nu K z-\cos \nu K z)\left(\nu \sin \nu K z_{0}-\cos \nu K z_{0}\right) I_{1}\left(\nu K r_{<}\right) K_{1}\left(\nu K r_{>}\right) \frac{\mathrm{d} \nu}{\nu^{2}+1}
\end{aligned}
$$

and $J_{1}, H_{1}, I_{1}$ and $K_{1}$ are Bessel functions of order one. 
The results in (24) and (27) are used to integrate (22) with respect to $s_{0}$ and give

$$
\psi\left(r_{0}, z_{0}\right)=-\int_{C} \phi(r, z) \frac{\partial}{\partial s} H\left(r, z ; r_{0}, z_{0}\right) \mathrm{d} s .
$$

(The constant of integration is zero from the choice of $\psi$ at infinity in (25).) Finally, integration by parts gives

$$
\psi\left(r_{0}, z_{0}\right)=\int_{C} \phi_{s}(r, z) H\left(r, z ; r_{0}, z_{0}\right) \mathrm{d} s .
$$

The point $\left(r_{0}, z_{0}\right)$ is moved onto $C$ and from (26) a first kind integral equation is obtained for the unknown function $\phi_{s}$ on $C$, namely

$$
\psi_{C}=\int_{C} \phi_{s}(r, z) H\left(r, z ; r_{0}, z_{0}\right) \mathrm{d} s, \quad\left(r_{0}, z_{0}\right) \in C,
$$

for some unknown arbitrary constant $\psi_{C}$. At this stage it is useful to employ the parametrisation of $C$ in terms of $t$ and write

$$
q(t)=\sigma(t) \phi_{s}(R(t), Z(t)), \quad 0 \leq t<2 \pi
$$

where $q(t)$ is a real-valued function, and use the shorthand notation

$$
H\left(t ; t_{0}\right) \equiv H\left(R(t), Z(t) ; R\left(t_{0}\right), Z\left(t_{0}\right)\right), \quad 0 \leq t, t_{0}<2 \pi
$$

Since we have assumed $\phi$ to be real with no loss of generality, $\psi$ and hence $\psi_{C}$ are also real and so the real and imaginary parts of (31) gives us a pair of coupled real equations

$$
\psi_{C}=\int_{0}^{2 \pi} q(t) H^{(r)}\left(t ; t_{0}\right) \mathrm{d} s, \quad 0 \leq t_{0}<2 \pi,
$$

and

$$
0=\int_{0}^{2 \pi} q(t) H^{(i)}\left(t ; t_{0}\right) \mathrm{d} s, \quad 0 \leq t_{0}<2 \pi
$$

where $H=H^{(r)}+\mathrm{i} H^{(i)}$.

The first of these two equations is written as

$$
(\mathcal{K} q)\left(t_{0}\right)=\psi_{C}
$$

where the operator $\mathcal{K}$ is defined by

$$
(\mathcal{K} q)\left(t_{0}\right)=\int_{0}^{2 \pi} q(t) H^{(r)}\left(t ; t_{0}\right) \mathrm{d} t
$$

and has a real symmetric kernel which is at most logarithmic singular. Continuity of $\phi$ on $C$ can be written as

$$
(q, 1)=0
$$


where the inner product notation

$$
(u, v)=\int_{0}^{2 \pi} u(t) v(t) \mathrm{d} t
$$

has been used for real functions $u, v \in L_{2}(0,2 \pi)$. Thus we require that (36) is solved for $q(t)$ subject to (38). In addition, in order to correspond to a trapped mode $q(t)$ must also satisfy (35). Now,

$$
H^{(i)}\left(r, z ; r_{0}, z_{0}\right)=\pi K r K r_{0} \mathrm{e}^{K\left(z+z_{0}\right)} J_{1}(K r) J_{0}\left(K r_{0}\right),
$$

and so (35) reduces to

$$
(q, f)=0
$$

where

$$
f(t)=K R(t) \mathrm{e}^{K Z(t)} J_{1}(K R(t))
$$

It is reasonable to ask whether a trapped mode defined to be the solution of (36), (38) and (41) ensures that (5) which is equivalent to (25) holds. By taking the limit $r_{0} \rightarrow \infty$ in (30) and adopting the appropriate form for $H$ in this limit, we find that the condition for the vanishing of the far field is precisely the condition (41) already required of the solution.

Once the function $q(t)$ has been determined, (30) may be used to give the value of the Stokes stream function (to within an arbitrary multiplicative constant) everywhere in the domain. Hence, from $(30)$

$$
\psi\left(r_{0}, z_{0}\right)=\int_{0}^{2 \pi} q(t) H^{(r)}\left(R(t), Z(t) ; r_{0}, z_{0}\right) \mathrm{d} t, \quad\left(r_{0}, z_{0}\right) \notin C
$$

since $\psi$ is real and, from (35), the imaginary part $H^{(i)}$ contributes nothing to the integral.

\section{Numerical procedure}

There is little chance of finding an explicit solution to the integral equation system (36), (38) and (41) and no further exact analytic progress appears possible. Accurate solutions to the integral equations must therefore be approximated using numerical methods. Since the integral equation system describing trapped modes makes no approximations to the governing equations or the boundary of the curve $C$, it is more in keeping with the formulation to expand the unknown function $q(t)$ in a set of functions having properties relating to the exact solution rather than using a simple discretisation method or a collocation scheme. 
Thus, following Porter (2001b) we will make an approximation to the unknown function $q(t)$ by writing

$$
q(t) \approx \tilde{q}(t)=\sum_{n=1}^{2 N} a_{n} u_{n}(t), \quad 0 \leq t<2 \pi
$$

where, for smooth curves $C$, it is sensible to choose

$$
\left.\begin{array}{c}
u_{2 n-1}(t)=\sin n t, \\
u_{2 n}(t)=\cos n t,
\end{array}\right\} \quad n=1, \ldots, N .
$$

(The omission of a $u_{0}=1$ term in the Fourier series ensures that (38) is satisfied.) The functions $u_{n}(t)$ are continuous, periodic and form a complete set. The set of coefficients $\left\{a_{n}\right\}$ are determined from a variational approximation to the solution of the integral equation in (36), equivalent to Galerkin's method, which is given by

$$
\left(\mathcal{K} \tilde{q}, u_{m}\right)= \begin{cases}2 \pi \psi_{C}, & m=0, \\ 0, & m=1, \ldots, 2 N .\end{cases}
$$

The equation which corresponds to $m=0$ may be thought of as determining $\psi_{C}$ and the remaining equations form the real homogeneous system

$$
\sum_{n=1}^{2 N} K_{m n} a_{n}=0, \quad m=1, \ldots, 2 N
$$

where

$$
K_{m n}=\left(\mathcal{K} u_{n}, u_{m}\right)=K_{n m}, \quad m, n \geq 1
$$

since $H^{(r)}\left(t ; t_{0}\right)$ is symmetric. Finally, the set $\left\{a_{n}\right\}$ must also satisfy the condition (41) with $q$ approximated by $\tilde{q}$, from which we obtain

$$
S \equiv \sum_{n=1}^{2 N} a_{n}\left(u_{n}, f\right)=0 .
$$

Thus, a non-dimensional trapped mode wave number is approximated by a value of $K a$ for which the smallest eigenvalue of the matrix $\lambda\left(K_{m n}\right)=0$, and the resulting eigenfunction satisfies the side condition (49).

Since $N$ is expected to be relatively small, most of the computational effort goes into the accurate computation of $K_{m n}$ which is given by

$$
K_{m n}=\int_{0}^{2 \pi} u_{m}\left(t_{0}\right) \int_{0}^{2 \pi} u_{n}(t) H^{(r)}\left(t ; t_{0}\right) \mathrm{d} t \mathrm{~d} t_{0},
$$


where

$$
\begin{gathered}
H^{(r)}\left(t ; t_{0}\right)=K R K R_{0}\left[\pi \mathrm{e}^{K\left(Z+Z_{0}\right)} J_{1}\left(K R_{<}\right) Y_{1}\left(K R_{>}\right)\right. \\
\left.+\frac{2}{\pi} \int_{0}^{\infty}(\nu \sin \nu K Z-\cos \nu K Z)\left(\nu \sin \nu K Z_{0}-\cos \nu K Z_{0}\right) \frac{I_{1}\left(\nu K R_{<}\right) K_{1}\left(\nu K R_{>}\right)}{1+\nu^{2}} \mathrm{~d} \nu\right] \\
R_{<}=\min \left\{R, R_{0}\right\}, \quad R_{>}=\max \left\{R, R_{0}\right\}
\end{gathered}
$$

and a shorthand notation has been employed whereby $R$ is written for $R(t)$ and $R_{0}$ for $R\left(t_{0}\right)$ etc. From (51) the integrand in (50) is inseparable and itself involves the evaluation of a semi-infinite integral. Furthermore it has logarithmic singularities along the line $t=t_{0}$ and at the two points defined by $2 \pi \pm\left(t-t_{0}\right)=0$. For efficient and accurate computation these singularities are first subtracted out from $H^{(r)}\left(t ; t_{0}\right)$ and integrated explicitly as detailed below.

From the asymptotic form of the modified Bessel functions (9.7.1 and 9.7.2 in Abramowitz \& Stegun (1965) ) the integrand in (51) behaves like

$$
L\left(t, t_{0} ; \nu\right)=\frac{\sin \nu K Z \sin \nu K Z_{0}}{2 \nu K R^{\frac{1}{2}} R_{0}^{\frac{1}{2}}} \mathrm{e}^{-\nu K\left|R-R_{0}\right|}, \quad \text { as } \nu \rightarrow \infty
$$

and from (3.947.1) in Gradshteyn \& Ryzhik (1980)

$$
\frac{2}{\pi} \int_{0}^{\infty} L\left(t, t_{0} ; \nu\right) \mathrm{d} \nu=\frac{1}{2 \pi K R^{\frac{1}{2}} R_{0}^{\frac{1}{2}}} \ln T\left(t, t_{0}\right)
$$

where

$$
T\left(t, t_{0}\right)=\left[\frac{\left(Z+Z_{0}\right)^{2}+\left(R-R_{0}\right)^{2}}{\left(Z-Z_{0}\right)^{2}+\left(R-R_{0}\right)^{2}}\right]^{\frac{1}{2}} .
$$

The function $T\left(t, t_{0}\right)$ is singular along the line $t=t_{0}$ and at the two points $2 \pi \pm\left(t-t_{0}\right)=0$, and it does not vanish in the domain of integration. Thus we follow Porter (2001b) and divide the computation of $K_{m n}$ into three parts, by writing

$$
K_{m n}=\int_{0}^{2 \pi} u_{m}\left(t_{0}\right) \int_{0}^{2 \pi} u_{n}(t) \hat{H}^{(r)}\left(t ; t_{0}\right) \mathrm{d} t \mathrm{~d} t_{0}+\left(K_{m n}^{(2)}+K_{m n}^{(3)}\right)
$$

where

$$
\begin{aligned}
& \hat{H}^{(r)}=K R K R_{0}\left[\pi \mathrm{e}^{K\left(Z+Z_{0}\right)} J_{1}\left(K R_{<}\right) Y_{1}\left(K R_{>}\right)\right. \\
& \left.+\frac{2}{\pi} \int_{0}^{\infty}(\nu \sin \nu K Z-\cos \nu K Z)\left(\nu \sin \nu K Z_{0}-\cos \nu K Z_{0}\right) \frac{I_{1}\left(\nu K R_{<}\right) K_{1}\left(\nu K R_{>}\right)}{1+\nu^{2}}-L\left(t, t_{0} ; \nu\right) \mathrm{d} \nu\right]
\end{aligned}
$$

and the matrices $K_{m n}^{(2)}$ and $K_{m n}^{(3)}$ are given by

$$
K_{m n}^{(2)}=\frac{K}{2 \pi} \int_{0}^{2 \pi} u_{m}\left(t_{0}\right) R_{0}^{\frac{1}{2}} \int_{0}^{2 \pi} u_{n}(t) R^{\frac{1}{2}} \ln \left[T\left(t, t_{0}\right) V\left(t, t_{0}\right)\right] \mathrm{d} t \mathrm{~d} t_{0}
$$


where

$$
V\left(t, t_{0}\right)=\left|t-t_{0}\right|\left(2 \pi-t+t_{0}\right)\left(2 \pi+t-t_{0}\right)
$$

and

$$
\begin{aligned}
K_{m n}^{(3)}= & -\frac{K}{2 \pi} \int_{0}^{2 \pi} u_{m}\left(t_{0}\right) R_{0}^{\frac{1}{2}} \int_{0}^{2 \pi}\left(u_{n}(t) R^{\frac{1}{2}}-u_{n}\left(t_{0}\right) R_{0}^{\frac{1}{2}}\right) \ln V\left(t, t_{0}\right) \mathrm{d} t \mathrm{~d} t_{0} \\
& -\frac{K}{2 \pi} \int_{0}^{2 \pi} u_{m}\left(t_{0}\right) u_{n}\left(t_{0}\right) R_{0}\left(\left(2 \pi+t_{0}\right) \ln \left(2 \pi+t_{0}\right)+\left(4 \pi-t_{0}\right) \ln \left(4 \pi-t_{0}\right)-6 \pi\right) \mathrm{d} t_{0} \cdot(60)
\end{aligned}
$$

The function $\hat{H}^{(r)}\left(t ; t_{0}\right)$ converges for all $0 \leq t, t_{0} \leq 2 \pi$ as does the integrand in $K_{m n}^{(2)}$. The logarithmic singularities which were originally in $H^{(r)}\left(t, t_{0}\right)$ have been integrated out explicitly in the second term in $K_{m n}^{(3)}$. Furthermore the integrals in both $K_{m n}^{(2)}$ and $K_{m n}^{(3)}$ are independent of the wave number $K$, and so they only need to be calculated once for each geometrical configuration.

\section{Results and discussion}

Numerical calculations are performed for a submerged elliptical torus whose boundary is parametrised by

$$
(r, z)=(c+a \cos t,-d+b \sin t), \quad 0 \leq t<2 \pi
$$

The method of Porter (2001a) is used to make computations of the transmission coefficient for a two-dimensional submerged ellipse with $b / a=0.16$ and $d / a=0.25$, and the results show that a zero of transmission exists at $K a=0.525$ and the plane wave approximation predicts that a torus with $c / a=3.600$ supports a trapped mode. Exact trapped modes are sought for an elliptical torus with the same values of $b / a$ and $d / a$ and neighbouring values of $c / a$ and $K a$. Figure 2 illustrates the curves $\lambda\left(K_{m n}\right)=0$ and $\tilde{S} \equiv \sum_{n=1}^{2 N} f_{n} \tilde{a}_{n}=0$ as functions of $c / a$ and $K a$ in the vicinity of $c / a=3.600$ and $K a=0.525$, where the coefficients $\left\{\tilde{a}_{n}\right\}$ are the coefficients in the eigenfunction for the eigenvalue of $K_{m n}$ which has the smallest magnitude. At the point where the two curves cross $\tilde{S}=S$ and so $\lambda\left(K_{m n}\right)=0$ and $S=0$ there. The two curves are generated by determining where real quantities change sign and the fact that the curves cross rather than touch gives confidence that a trapped mode exists, even though the values of $K a$ and $c / a$ may not be found exactly. The trapped mode values for this configuration, computed using the integral equation method with $N=8$, are given by

$$
\frac{c}{a}=3.62155, \quad K a=0.52627
$$




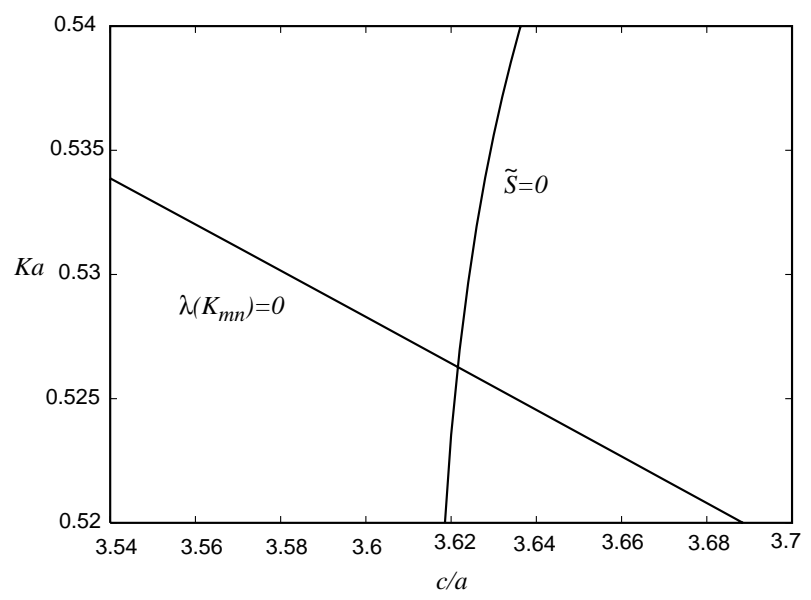

Figure 2: Crossing of the curves $\lambda\left(K_{m n}\right)=0$ and $\tilde{S}=0$ for an elliptical torus, $b / a=0.16, d / a=0.25$.

Table 1 shows the convergence of the scheme as $N$ increases. The accuracy of the numerical quadrature (160 evaluations of the integrand per integral) was also increased with no change in the five decimal places quoted here. The results from the plane wave approximation are within $1 \%$ of those predicted by this exact formulation.

\begin{tabular}{|c|c|c|}
\hline$N$ & $K a$ & $c / a$ \\
\hline 4 & 0.52780 & 3.63018 \\
5 & 0.52679 & 3.62042 \\
6 & 0.52631 & 3.62176 \\
7 & 0.52628 & 3.62156 \\
8 & 0.52627 & 3.62155 \\
\hline
\end{tabular}

Table 1: Convergence of $K a$ and $c / a$ with truncation size $N$ for an elliptical torus with $b / a=0.16$ and $d / a=0.25$

A number of computed results for trapped mode values of $K a$ and $c / a$ for different values of $b / a$ and $d / a$ are summarised in table 2 . These are all computed from the exact formulation but in each case the values from the plane wave approximation were used to determine the region of parameter space in which to look for the trapped modes. A null entry signifies that there are no trapped modes found for that particular combination of parameter. The absence of results in the lower triangle are explained simply by the fact that we must choose $b / a<d / a$ for the torus to be submerged. The upper triangle of no trapped modes indicates that the more deeply submerged the top of the torus is, the longer the horizontal extent of the cross-section has to be for trapped 
modes to exist. This is confirmed by the results in figure 3 where we present the locus of values of $K a$ and $c / a$ as $b / a$ is varied for various values of $d / a$. These curves are similar to those presented in Porter (2001b).

\begin{tabular}{|c|c|c|c|c|c|c|}
\hline \multirow[b]{2}{*}{$d / a$} & \multicolumn{6}{|c|}{$b / a$} \\
\hline & 0.04 & 0.08 & 0.12 & 0.16 & 0.2 & 0.24 \\
\hline \multirow{2}{*}{0.3} & \multirow{2}{*}{ - } & \multirow{2}{*}{ - } & \multirow{2}{*}{-} & \multirow{2}{*}{-} & 4.22430 & 3.98209 \\
\hline & & & & & 0.48159 & 0.45113 \\
\hline \multirow{2}{*}{0.25} & \multirow{2}{*}{ - } & 3.96860 & 3.71152 & 3.62155 & 3.66547 & 4.21728 \\
\hline & & 0.56043 & 0.56145 & 0.52627 & 0.45148 & 0.28664 \\
\hline \multirow{2}{*}{0.2} & 3.25850 & 3.26333 & 3.33559 & 3.57864 & \multirow{2}{*}{ - } & \multirow{2}{*}{-} \\
\hline & 0.65717 & 0.61093 & 0.53944 & 0.42465 & & \\
\hline \multirow{2}{*}{0.15} & 3.03694 & 3.23079 & 3.75368 & \multirow{2}{*}{-} & \multirow{2}{*}{-} & \multirow{2}{*}{ - } \\
\hline & 0.63019 & 0.52534 & 0.36853 & & & \\
\hline \multirow{2}{*}{0.1} & 3.31776 & 4.43568 & \multirow{2}{*}{-} & \multirow{2}{*}{ - } & \multirow{2}{*}{ - } & \multirow{2}{*}{-} \\
\hline & 0.47983 & 0.27962 & & & & \\
\hline
\end{tabular}

Table 2: Summary of values of $c / a$ (upper entry) and $K a$ (lower entry) for trapping by an elliptical torus for selected values of aspect ratio, $b / a$, and submergence, $d / a$.

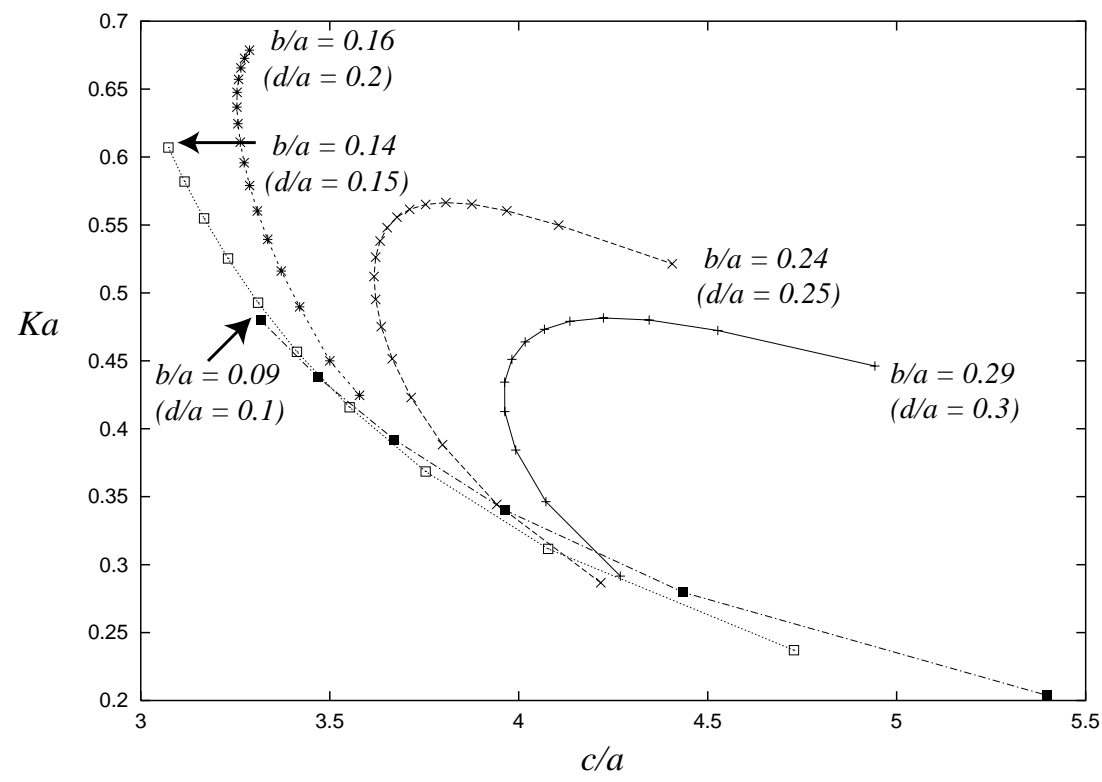

Figure 3: Locus of trapped modes for elliptical cross-section as $b / a$ varies for various fixed values of $d / a$. Values of $b / a$ decrease in steps of 0.1 from point to point along each of the curves. 
Once trapped mode values of $K a$ and $c / a$ have been found for a particular torus geometry, the corresponding approximation $\tilde{q}(t)$ to $q(t)$ can be used in (43) to obtain the value of the Stokes stream function everywhere in an axial plane. In figure 4 we plot a cross-section through the stream surfaces at trapping for the submerged elliptical torus with $b / a=0.16, d / a=0.25$. From the figure it is clear that these stream surfaces also correspond to those for a surface-piercing torus which surrounds the submerged elliptical torus and connects the free surface. Apart from the region above the submerged elliptical torus, the stream surface pattern is very similar to that obtained for the toroid described in McIver \& McIver (1997). The flow represents a sloshing backwards and forwards in the radial direction, in a region above the body of torus which is bounded by stream surfaces which connect the surface of the torus at a line of stagnation points, to the mean free surface. A natural question to ask is whether the stream surfaces may be continued analytically into the interior of the torus and a second closed stream surface formed which represents another submerged body for the same flow. However, such a construction would mean that there existed a continuous, non-constant velocity potential in the region between two fixed rigid boundaries and a simple energy argument shows that this is not possible.

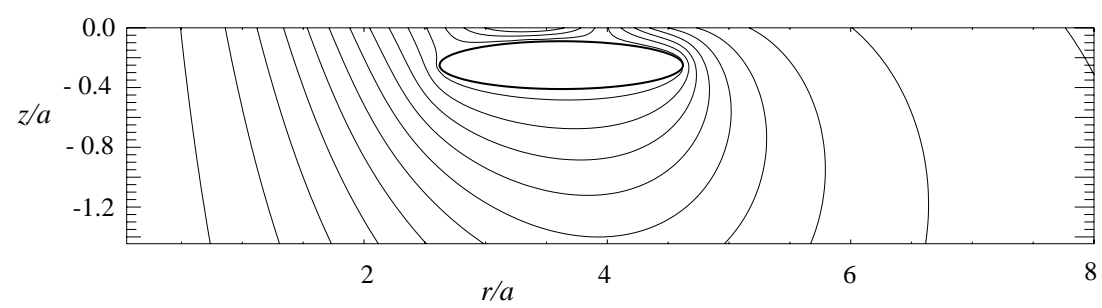

Figure 4: The stream surfaces of the flow in an axial plane at trapping for a submerged elliptical torus (thick line). Here $b / a=0.16, d / a=0.25, K a=0.52677, c / a=3.62155$.

As was noted in the introduction, the existence of a trapped mode means that the solution to any forced problem for the torus is not unique and may not exist at the trapped mode frequency. Suppose that the torus is forced to oscillate in heave at the trapped mode wave number $K_{0}=\omega_{0}^{2} / g$. If the heave potential $\operatorname{Re}\left[\phi_{h} \mathrm{e}^{-\mathrm{i} \omega_{0} t}\right]$ exists it must satisfy $(1)-(3)$, a radiation condition and

$$
\frac{\partial \phi_{h}}{\partial n}=n_{z}, \quad \text { on } \quad S,
$$

where $n_{z}$ is the component of the inward normal to the torus in the $z$ direction. An application of Green's theorem to $\phi_{h}$ and the trapped mode potential $\phi_{0}$ shows that a necessary condition for $\phi_{h}$ to exist is that

$$
\int_{C} r \phi_{0} n_{z} \mathrm{~d} s=\int_{0}^{2 \pi} \frac{R(t) \phi_{0}(t) R^{\prime}(t)}{\sigma(t)} \frac{\mathrm{d} s}{\mathrm{~d} t} \mathrm{~d} t=\int_{0}^{2 \pi} \phi_{0}(t) \frac{\mathrm{d}}{\mathrm{d} t}\left[\frac{R^{2}(t)}{2}\right] \mathrm{d} t=0 .
$$


Integration by parts transforms this condition into

$$
\int_{0}^{2 \pi} q(t) R^{2}(t) \mathrm{d} t=0
$$

as $\phi_{0}$ is continuous on $C$. The expansion for $q(t)$ in (44) and the expression for $R(t)$ in $(61)$ are substituted in (65) and the orthogonality of the trigonometric functions is used to show that the necessary condition for the existence of $\phi_{h}$ reduces to

$$
a_{4}+\frac{4 c}{a} a_{2}=0
$$

Calculations are made for elliptical toruses with a range of quadruples $(b / a, d / a, c / a, K a)$, including $(0.16,0.25,3.62155,0.52627)$ and in no case is this condition satisfied. Thus the heave potential does not exist at the trapped mode frequency for these bodies. Supporting evidence for this conclusion is presented in figures 5 and 6 , where the real and imaginary parts of the vertical force on the torus, the heave added mass $a_{33}$ and damping $b_{33}$, nondimensionalised by $\rho V$ and $\rho V \omega$ respectively where $\rho$ is the water density and $V$ is the volume of the torus, are plotted for a torus with $b / a=0.16, d / a=0.25$ and $c / a=3.62155$. The calculations were made using the boundary element code WAMIT, with a varying number of panels. Experimentation showed that the best distribution of panels is at equal azimuthal angles and at equal values of the arc length around the boundary of the torus. The iterative solver option on WAMIT did not give convergence near the trapped mode frequency and so all the calculations have been performed using direct Gaussian elimination.

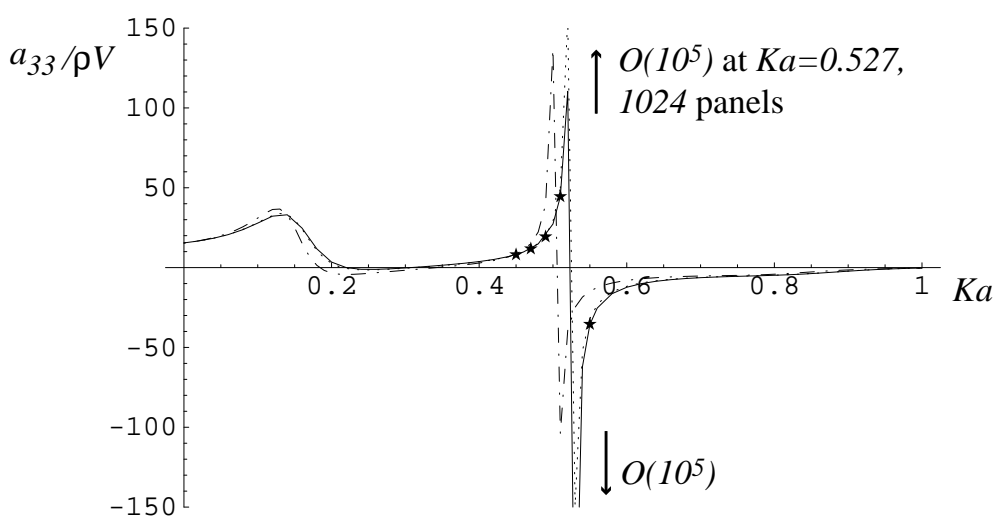

Figure 5: Heave added mass for a submerged elliptical torus, $b / a=0.16, d / a=0.25$, $c / a=3.62155$. Number of panels on $1 / 4$ torus, $\star: 1920,-: 1024, \cdots: 512,-\cdot-: 256$

The figures for the added mass and damping show the same type of singular behaviour that was found by Newman (1999) for the surface-piercing toroid. Although there are no possible irregular frequency effects as the torus is submerged, there is a clear broad band singularity in the 


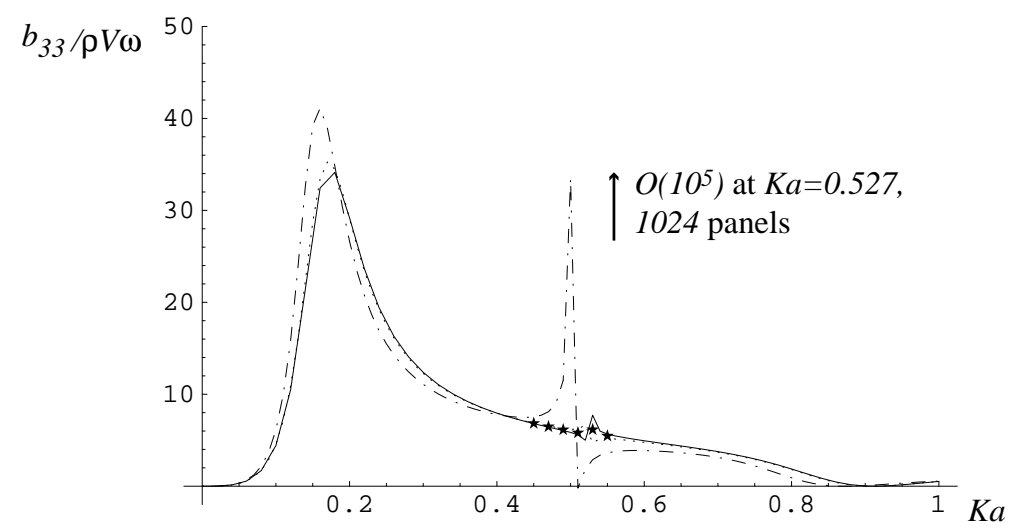

Figure 6: Heave damping for a submerged elliptical torus, $b / a=0.16, d / a=0.25$, $c / a=3.62155$. Number of panels on $1 / 4$ torus, $\star: ~ 1920,-: 1024, \cdots: 512,-\cdot-: 256$

added mass and a much narrower band singularity in the damping in the vicinity of the trapped mode frequency.

It is possible to show that the amplitude of the fluid oscillation grows without bound when the torus is forced to oscillate in heave actually at the trapped mode frequency $\omega_{0}$. (This is analogous to an undamped mass-spring system which undergoes resonance when forced at its natural frequency.) A time domain problem is considered in which the torus starts to make small vertical oscillations at $t=0$ in such a way that the time dependent velocity potential $\Phi(r, z, t)$ satisfies

$$
\frac{\partial \Phi}{\partial n}=V n_{z} \sin \omega_{0} t H(t) \quad \text { on } S,
$$

where $V$ is a constant and $H(t)$ is the unit step function, and the initial conditions are $\Phi(r, 0,0)=$ $0, \partial \Phi / \partial t(r, 0,0)=0$. An application of Green's theorem to $\Phi(r, z, t)$ and the potential $\phi_{0}(r, z) \cos \omega_{0} t$, where $\phi_{0}$ is the trapped mode potential, followed by two integrations in time shows that

$$
\int_{0}^{\infty} \phi_{0}(r, 0) \Phi(r, 0, t) r \mathrm{dr}=\frac{\mathrm{Vg}}{2 \omega_{0}^{2}}\left(\sin \omega_{0} \mathrm{t}-\omega_{0} \mathrm{t} \cos \omega_{0} \mathrm{t}\right) \int_{\mathrm{C}} \phi_{0} \mathrm{rn} \mathrm{z}_{\mathrm{z}} \mathrm{ds}
$$

where the governing equation, the linearised time dependent free surface condition and the far-field behaviour of the potentials have been used. As it has already been shown numerically that the necessary condition for the heave potential to exist is not satisfied, $\int_{C} \phi_{0} r n_{z} \mathrm{~d} s \neq 0$. Thus there is a term in $\Phi$ (its projection in the direction of the trapped mode) which represents a growing oscillation at the trapped mode frequency. 


\section{Conclusion}

Strong numerical evidence has been presented which shows that surface gravity waves may be trapped by three-dimensional submerged obstacles. A plane wave approximation was used to predict the trapping of waves by a submerged torus at a frequency at which the two-dimensional cross-section of the torus had a zero of transmission. This led to the consideration of a submerged elliptical torus as a range of ellipses of varying aspect ratio and submergence depths do not transmit waves at isolated frequencies. An integral equation and side condition which the exact trapped mode must satisfy were derived and numerical calculations were made of the size of torus which could support a trapped mode. A necessary condition for the existence of the heave potential at the trapped mode frequency was derived and it was shown that this condition was not satisfied numerically for the bodies under consideration. This conclusion was supported by the calculation of the heave added mass and damping over a range of frequencies, as these displayed a singular behaviour in the vicinity of the trapped mode frequency. An analysis of the initial value problem for a torus undergoing vertical oscillations at the trapped mode frequency showed that the potential contains a term which represents an oscillation which grows with time.

A natural extension of the work would be to consider whether non-axisymmetric trapped modes may be supported by a submerged torus. Kuznetsov \& McIver (1997) gave numerical evidence that modes with a $\cos n \theta$ variation may be trapped by certain surface-piercing toruses, where $\theta$ is the azimuthal angle and the plane wave approximation predicts that such modes exist for both a submerged and a surface-piercing torus of suitable radius, provided that its crosssection supports a zero of transmission. However, the numerical method outlined in this work, although particularly convenient to use for the location of axisymmetric modes, cannot be used to locate non-axisymmetric modes because the integral equation formulation relied on the existence of Stokes stream function for the flow.

\section{Acknowledgement}

Part of this work was supported by EPSRC grant GR/M30937

\section{References}

[1] Abramowitz, M. And Stegun, I. A. 1965. Handbook of Mathematical Functions, Dover 
Publications, New York

[2] Evans, D. V. \& Porter, R. 1998. An example of non-uniqueness in the two-dimensional linear water-wave problem involving a submerged body Proceedings Royal Society London A 454, $3145-3165$.

[3] Gradshteyn, I.S. \& Ryzhik, I. M. 1980. Table of Integrals, Series and Products, Academic Press, London

[4] Hulme, A. 1983. A ring source/integral/equation method for the calculation of hydrodynamic forces exerted on floating bodies of revolution Journal of Fluid Mechanics 128, 387-412.

[5] Kuznetsov, N. G. \& McIver, P. 1997. On uniqueness and trapped modes in the waterwave problem for a surface-piercing axisymmetric body Quarterly Journal of Mechanics and Applied Mathematics 50, 565-580.

[6] Kuznetsov, N. G. McIver, P. \& Linton, C. M. 2001. On uniqueness and trapped modes in the water-wave problem for vertical barriers Wave Motion 33, 283-307.

[7] Linton, C. M. \& Kuznetsov, N. G. 1997. Non-uniqueness in two-dimensional water-wave problems: numerical evidence and geometrical restrictions Proceedings Royal Society London A 453, 2437-2460.

[8] McIver, M. 1996. An example of non-uniqueness in the two-dimensional linear water wave problem Journal of Fluid Mechanics 315, 257-266.

[9] McIver, M. 2000. Trapped modes supported by submerged obstacles Proceedings Royal Society London A 456, 1851-1860.

[10] McIver, P. \& McIver, M. 1997. Trapped modes in an axisymmetric water-wave problem Quarterly Journal of Mechanics and Applied Mathematics 50, 165-178.

[11] McIver, P. \& Newman, J. N. 2001. Non-axisymmetric trapping structures in the threedimensional water-wave problem Proceedings 16th International Workshop on Water Waves and Floating Bodies, Hiroshima, Japan.

[12] Newman, J. N. 1977. The motion of a floating slender torus Journal of Fluid Mechanics 83, 721-735. 
[13] Newman, J. N. 1999. Radiation and diffraction analysis of the McIver toroid Journal of Engineering Mathematics 35, 135-147.

[14] Porter, R. 2001a. Scattering of surface waves by submerged cylinders of arbitrary crosssection. submitted to Proceedings Royal Society London A

[15] Porter, R. 2001b. Trapping of waves by pairs of submerged cylinders submitted to Proceedings Royal Society London A

[16] Simon, M. J. 1982. Multiple scattering in arrays of axisymmetric wave-energy devices. Part 1. A matrix method using a plane-wave approximation Journal of Fluid Mechanics 120, 1-25.

[17] Simon, M. J. \& URSell 1984. Uniqueness in linearized two-dimensional water-wave problems Journal of Fluid Mechanics 148, 137-154. 